\title{
THE HARP PLASMA EXPERIMENT ON-BOARD THE PHOBOS 2 SPACECRAFT: PRELIMINARY RESULTS
}

\author{
P. KIRALY, R. LOCH, K. SZEGÖ, I. SZEMEREY, I. T, SZÜCS and M. TATRALLYAY \\ Central Research Institute for Physics, PO Box 49. H-1525 Budapest. Hungary \\ N. M. SHUTTE, A. V. DYACHKOV, K. I. GRINGAUZ, S. SHERONOVA and M. I. VERIGIN \\ Space Research Institute. U.S.S.R. Academy of Sciences, Profsoyuznaya 84/32, \\ Moscow 117810, U.S.S.R. \\ and \\ T. E. CRAVENS,* T. I. GOMBOSI, A. F. NAGY and W. SIIARP \\ University of Michigan, Ann Arbor, MI 48109-2143, U.S.A.
}

(Received 28 June 1990)

\begin{abstract}
The HARP differential electrostatic analyzer measured thermal and suprathermal electron and ion fluxes and distributions in the Mars environment. High bit-rate data obtained around the pericenter passage on elliptical orbits early in February 1989 will be highlighted, and correlation with results of other experiments will be discussed. A similarity between the HARP total count rate variations and the spacecraft potential measurements of the Plasma-Wave System experiment is established. The variation of electron energy spectra is discussed in various regions of the solar wind interaction with Mars. Tentative results on electron and ion anisotropy are presented, making use of regular variations of the ratio of count rates of two sensor heads looking in perpendicular directions. Magnetic field data of the MAGMA magnetometer are used for comparison.
\end{abstract}

\section{INTRODUCTION}

The HARP hyperbolic electrostatic analyzer aboard the Phobos 2 spacecraft measured fluxes, angular distributions, and energy spectra of thermal and suprathermal electrons and ions in the Martian environment. This survey of preliminary results will focus upon the description of data collected during the periods of high bit-rate telemetry regime at the first and second close encounters with Mars on 1 and 4/5 February 1989, respectively. For those periods the quality and time coverage of HARP data allow comparison with data obtained by other plasma and field experiments. The present report extends the scope of the short summary of HARP results published in the special Phobos 2 issue of Nature (Shutte et al., 1989). An accompanying paper by Shutte et al. (1991) in the present special issue of Planetary and Space Science on Phobos-Mars discusses the observed spectra and their physical implications in more detail.

For a detailed description of the instrument and some discussion of its acceptance characteristics, we refer the reader to the HARP technical paper (Szücs et al., 1990). Here we describe only those aspects which are absolutely necessary.

*Also at University of Kansas, Lawrence, KS 66045. U.S.A.
The HARP sensor system consists of two sensor heads arranged at right angles to each other. The field of view of each head is split into four viewing sectors covering a field of view of $20^{-} \times 10^{-1}$ each. The eight viewing sectors of the sensor system are arranged like a fan centered on the anti-solar direction and cover approximately half a circle, the plane of the fan being perpendicular to the ecliptic in periods when Phobos 2 is in the three-axes stabilized mode. The HARP device was mounted on the antisolar side of the solar panel as can be seen in the sketch given in Fig. 1 of Sagdeev and Zakharov (1989), describing the arrangement of scientific instruments aboard Phobos 2. Parameters of the elliptical orbits are given in the same paper. A graphical presentation of the second elliptical orbit with the positions of the relevant plasma regions is also shown by Shutte et al. (1991) in the present issue.

During the first two encounters with Mars, the spacecraft was rotating with a $10-\mathrm{min}$ period around an axis slightly offset relative to the solar-antisolar direction. As will be discussed later, this rotation led to a periodic variation in the ratio of count rates of the two sensor heads.

During the first two Mars encounters, 25 energy steps were chosen for electrons $(3.4-550 \mathrm{eV})$ and 50 for ions $(0.25-550 \mathrm{eV})$ with a measurement time of $1 \mathrm{~s}$ for each step. Out of the four viewing sectors of 
each sensor head, two (the so-called inner sectors which are closer to the symmetry axis of the head) performed much better, as discussed by Szücs et al. (1990). In the present paper, only data measured in those sectors will be discussed.

The effective energy-geometric factor of the inner sectors is $1.3 \times 10^{-6} \mathrm{~cm}^{2} \mathrm{sr} \mathrm{eV} \mathrm{eV}-1$, while that of the other ones is only $2.4 \times 10^{-7} \mathrm{~cm}^{2} \mathrm{sr} \mathrm{eV} \mathrm{eV}-1$. These low values make the instrument insensitive for detailed study of typical solar wind energy distributions. The situation is somewhat better in the Mars environment where total count rates can reach several thousand. It should be remembered, however, that the HARP is best suited for measurements in the ionosphere of both Earth and Mars. Since the pericenter altitude of the Phobos 2 spacecraft was higher than planned, the full potentialities of the instrument could not be exploited.

\section{VARIATIONS OF TOTAL COUNT RATES AND ENERGY SPECTRA}

The HARP data can be presented as time sequences of two-dimensional numerical arrays $(4 \times 25$ count rates for electrons, $4 \times 50$ for ions, if we consider only the inner angular sectors as discussed above). To emphasize different aspects, it is sometimes useful to take the sum of the data over directions, or energies, or both, and to consider the time variation of such data sets. The total count rates for electrons and ions can be considered as such a compressed measure of electron and ion fluxes seen by the instrument as a whole. As shown in our earlier paper (Shutte et al., 1989), the variation of total count rates with time can be used for separating some plasma regions in the planetary environment. The additional information about energy spectra and angular distributions should of course refine the crude, preliminary information provided by the total count rates.

During the high bit-rate telemetry periods, there was one complete measurement cycle per $90 \mathrm{~s}$. In spite of this relatively poor time resolution, the variation of total count rates clearly shows some of the characteristic features of the SW/Mars interaction regions also seen by other instruments. Figures 1 and 2 show the HARP total count rates for electrons and ions (represented in arbitrary units), together with the time dependence of the spacecraft potential as measured by the Langmuir probe of the Plasma-Wave System and kindly provided by Dr R. Grard (1989). Note that the positive axis is downward-directed for the spacecraft potential. A short description of the PlasmaWave System can be found in Grard et al. (1989). The

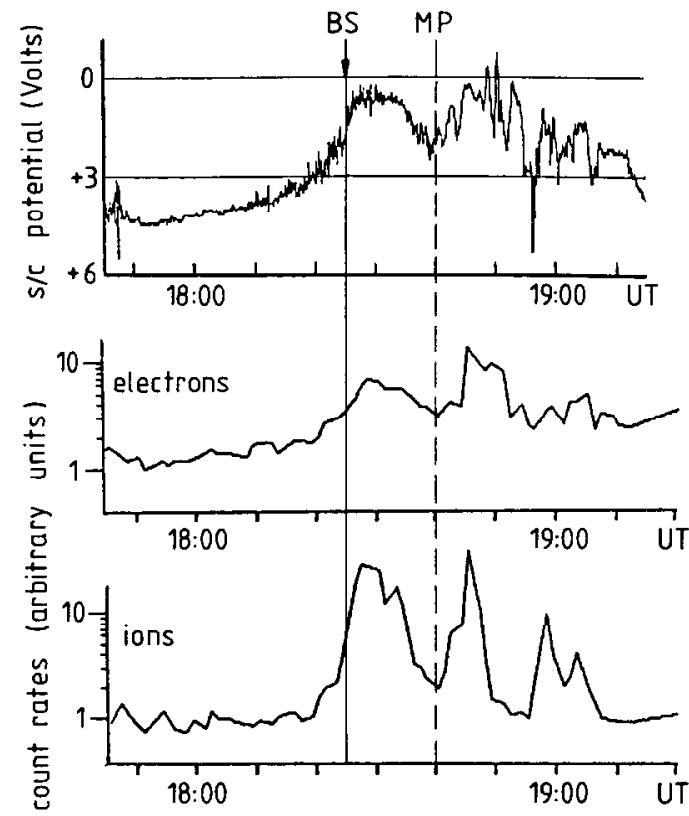

Fig. I. TIME SEQUENCE OF SPACECRAFT POTENTIAL, AND TOTAL COUNT RATES FOR ELECTRONS AND IONS DURING THE FIRST Mars enCOUNTER ON 1 FEbruary 1989.

The lime of the bow shock (BS) and the miagnetopause (MP) crossing is indicated.

comparison was restricted to those periods where both sets of data were available.

The neutralization of the spacecraft during the encounter is a consequence of the increased fluxes. Far from the planet, the positive spacecraft potential is caused by the escape of photoelectrons. In the solar wind, typically a potential difference of several volts is needed for a sufficient compensation current to reach the spacecraft from the surrounding plasma. In the denser plasma of the magnetosheath and magnetosphere, a much smaller equilibrium potential difference is expected and observed. A good correlation of the HARP count rate enhancements with the variation of the spacecraft potential (particularly during the first encounter) is clearly seen.

A comparison of the electron and ion curves shows several similarities, but also significant differences. One important difference is that the variations are more pronounced in the ion fluxes. This may be partly connected to the difference in energy coverage of HARP on the low energy side ( $>3.4 \mathrm{eV}$ for electrons, $>0.25 \mathrm{eV}$ for ions). A cold electron component-even if it is present - is not detected, while a substantial flux of cold ions is seen mainly in the magnetosheath and tail regions of the trajectory. The count rates of both 


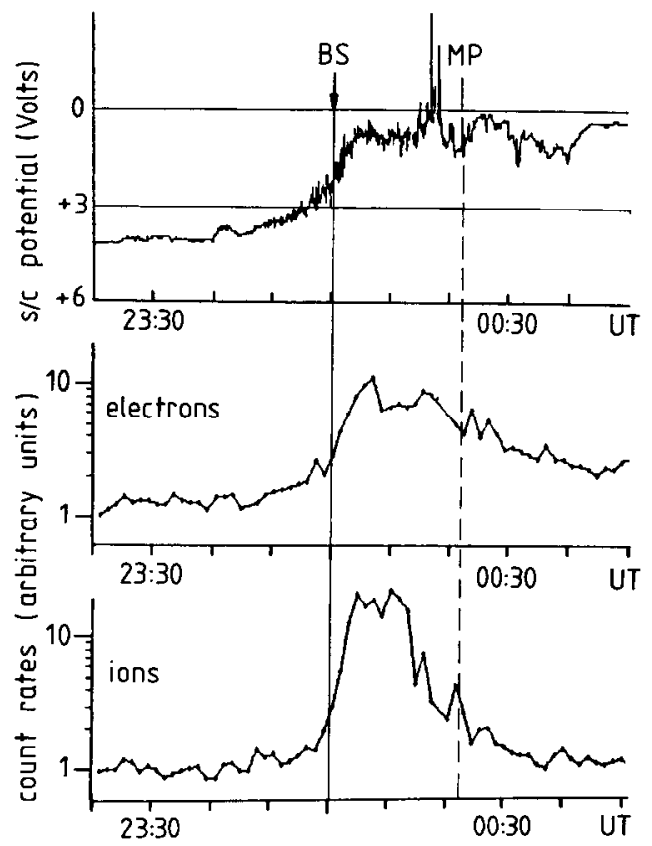

Fig. 2. TimE SEQUENCE OF SPACECRAFT POTENTIAL, AND tOTAL COUNT RATES FOR ELECTRONS AND IONS DURING THE SECONI) MARS ENCOUNTER ON 4/5 February 1989.

The time of the bow shock (BS) and the magnetopause (MP) crossing is indicated.

electrons and ions increase in the last $10-15 \mathrm{~min}$ before the bow shock crossing (indicated by a continuous vertical line). The increase becomes steeper before reaching the shock, and this steep increase continues after the shock crossing. A broad intensity peak with some fluctuation and eventual decrease characterizes the magnetosheath region. The variations are larger in ion fluxes than in electrons. After crossing the magnetopause (indicated by dashed line). strong variations are detected at the first encounter, while a relatively smooth behavior is observed during the second one. Inside the magnetosphere, ion count rates drop back to the pre-shock level fairly soon, while for electrons a higher level is maintained (this is better seen in Fig. 2). In the tail region following the second encounter (which is not covered by Fig. 2), strong fluctuations were found in the electron count rates.

Now we turn our attention to the time variation of energy spectra. The distribution of count rates over energy channels is easy to translate into energy spectra. The differential intensity $j(E)$ is obtained from count rates $C_{E}$ by using the simple formula $j(E)=C_{E} / G E$ where $G$ is the energy-geometric factor given in the Introduction, and the factor $E$ takes into account the fact that the width of the energy channels is proportional to energy. In a stacked plot representation of the time variation of spectral features, however, the division by $E$ usually makes the picture less clear, particularly when small counts are divided by small $E$ values, thus enhancing fluctuations and causing frequent crossovers of lines. In order to avoid the blurring of the picture on the left-hand side (i.e. for small energies), we use uncorrected sequences of count rates (loosely referred to as spectra). Some characteristic electron spectra corrected for the energy dependence of the width of energy channels are presented and discussed in an accompanying paper (Shutte et al., 1991). Further discussions will be restricted to electrons.

The variation of uncorrected electron spectra during the high bit-rate telemetry periods of the first and second encounters is shown in Figs 3 and 4, respectively. Some smoothing in both energy and in time (three-point sliding average) and a subsequent background subtraction (one per energy channel) have been applied. The results are shown separately for the four viewing sectors. Times for the bow shock and magnetopause transitions, closest approach to the planet (at an altitude of about $860 \mathrm{~km}$ ) and-for the second encounter only - the entry into the eclipse cone of Mars are indicated. Electron spectra do not show any sharp boundary between the magnetosheath and the magnetosphere. The magnetopause (MP) is indicated in Figs 3 and 4 as it was reported by different teams at the Lindau meeting in May 1989. A tentative identification of spectral features with physical domains of the interaction of the solar wind with Mars has already been presented by Shutte ef ol. (1989). Here we only add some comments and extend the list of those features.

In Fig. 3, some enhancements extending to the highest energies are seen around 17:45 U.T. (PH) when Phobos 2 came near to the orbit of the Phobos moon. This feature appears to corroborate the claims of several groups (Riedler et al., 1989; Grard et al., 1989 ; Dubinin et al. 1990) for some disturbance possibly associated with a plasma or dust torus. However, alternative explanations (e.g. magnetic connection to the bow shock) cannot be excluded. In Fig. 4, spectra are slightly disturbed close to the Phobos orbit.

In the last 10-15 min before the bow shock crossing, enhanced total count rates were observed as already mentioned above. Figures 3 and 4 show the doublepeaked spectral structure of those enhancements. with the warm component becoming hotter and more pronounced as the bow shock is approached. The relationship between these upstream electron spectra and wave activity observed by the Plasma-Wave System is discussed by Sagdeev et al. (1990). 


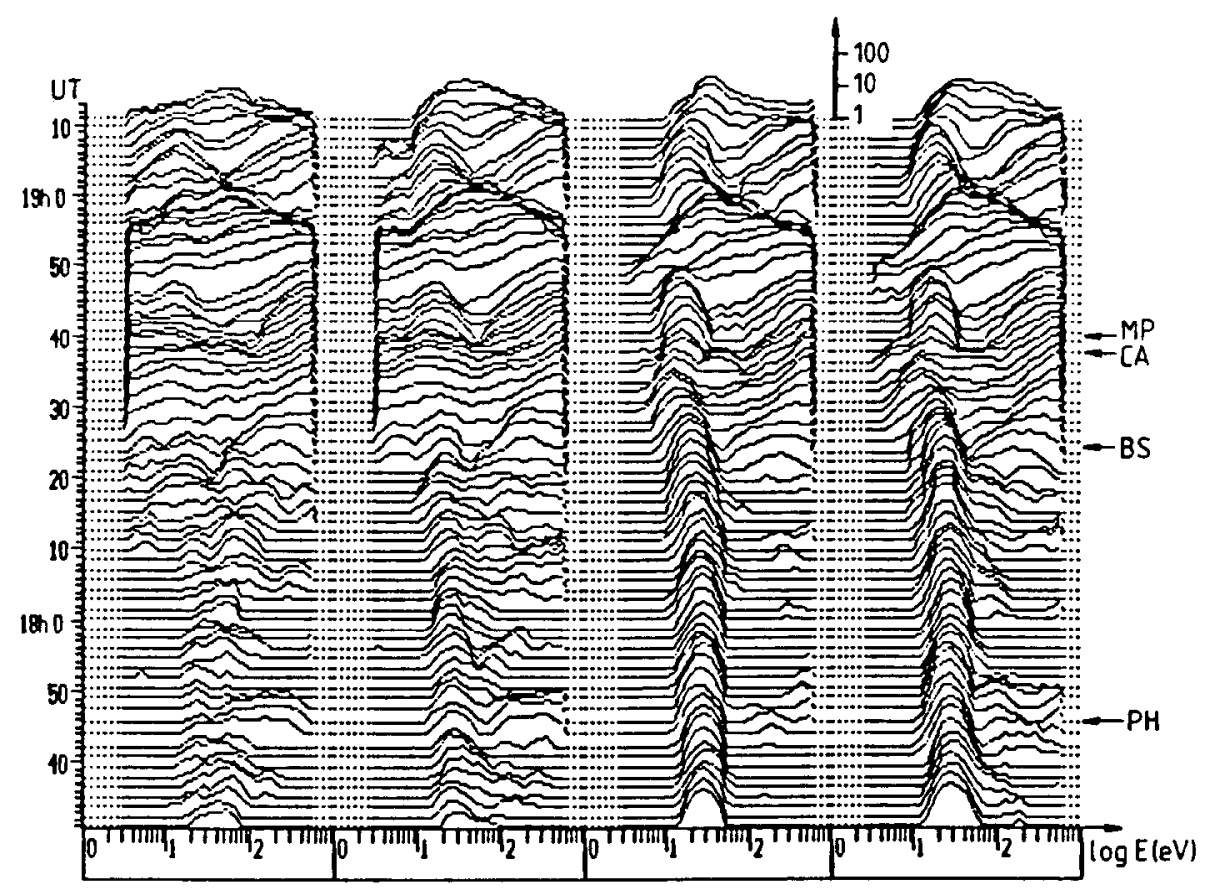

Fig. 3. TIME VARIATION OF THE ENERGy SPECTRA OF ELECTRONS MEASURED By FOUR VIEWING SECTORS OF IHE HARP deVICE dURING 'THE FIRSI MARS ENCOUNTEK UN 1 FEbruaky 1989.

The transition time of the Phobos moon orbit (PH), bow shock (BS), magnetopause (MP), and closest approach (CA) is indicated.

In the magnetosheath, spectra clearly extend beyond the $550 \mathrm{eV}$ upper limit of our energy range. This is also true for some time intervals after crossing the magnetopause at the first encounter. It looks as if the spacecraft returned to the magnetosheath after a magnetospheric section, and entered the magnetosphere again at about 19:05 U.T. (see Fig. 3). The single-peaked warm spectra seen here are very similar to those observed during the second encounter between the magnetopause and the tail boundary (see Fig. 4). The tail spectra of the second encounter remind one of those seen in the magnetosheath, with the important difference that in the tail region the lowenergy peak is less pronounced or missing altogether. The good correlation of the appearance of these spectra in the tail region with the detection of heavy ion fluxes by the TAUS ion spectrometer shows that this region corresponds to the plasma sheet (Shutte et al., 1989, 1991).

\section{INDICATION OF ANISOTROPY}

As mentioned in the Introduction, during the first two encounters discussed here, Phobos 2 was rotating, thus the two perpendicular sensor heads of HARP regularly changed their orientation. As a first approximation, we suppose that the rotation axis coincides with the $x$ axis of the spacecraft. This axis is parallel to the Sun-spacecraft line both in the rotating and the three-axes stabilized state. The velocity vector of the solar wind is also approximately parallel to this line. The central directions of the field of view of the two sensor heads then enclose $45^{\circ}$ with the axis of rotation, and as the spacecraft rotates, they sweep around as opposite generants of the same cone. Periodic variations of the count rates (or of the ratio of the two count rates) are expected if the particle distributions are anisotropic, and the anisotropy has a component perpendicular to the Sun-spacecraft line. It appeared worthwhile to check whether any such periodicity is observed in the ratio of count rates of the two sensor heads.

Figures 5 and 6 show the results for the first and second encounters, respectively. For comparison, some MAGMA magnetometer data kindly provided by Dr K. Schwingenschuh (1989) are also displayed. The magnetometer measured the magnetic field components in the rotating frame of reference of 


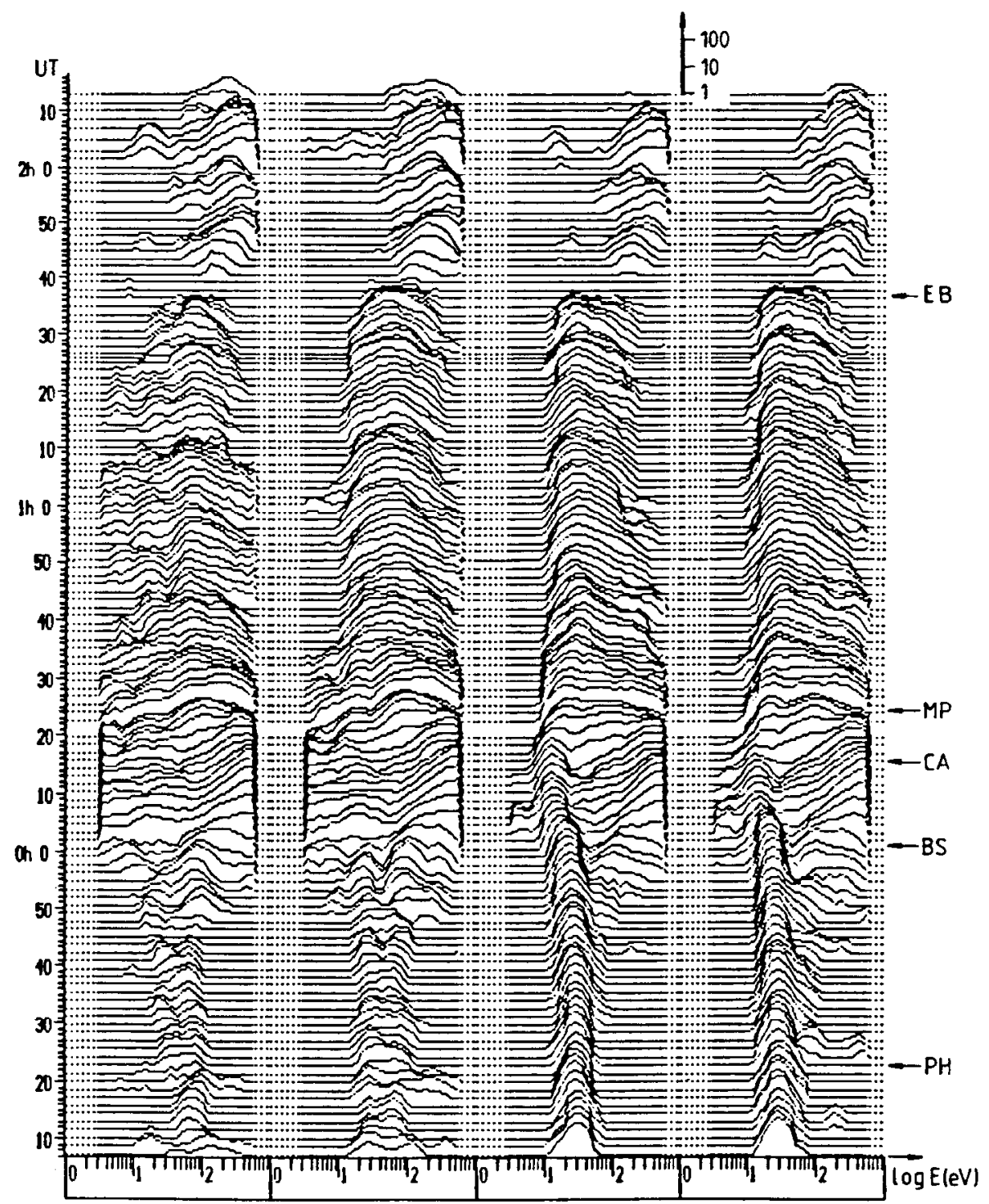

Fig. 4. Time VARIATION OF THE ENERGy SPECTRA OF ELECTRONS MEASURED BY FOUR VIEWING SECTORS OF THE HARP DEVICE DURING THE SECOND MARS ENCOUNTER ON 4/5 FEBRUARY 1989.

The transition time of the Phobos moon orbit (PH), bow shock (BS), magnetopause (MP), closest approach (CA), and eclipse boundary (EB) is indicated

the spacecraft, thus a constant magnetic field vector appears to be rotating. The bottom curve shows that the modulus of the magnetic field is practically constant in the upstream region, justifying the assumption that practically the entire variation of the $B_{z}$ component is due to rotation. The variation of the $B_{y}$ component not displayed here is similar to that of $B$, but shifted by a quarter of the rotation period, while $B_{x}$ varies much less due to the approximate coinci- dence of the rotation axis with the spacecraft $x$ axis. In the shock and the downstream region, the magnetic field varies on a multitude of time-scales, making the magnetic field data less suitable to characterize rotation. The first results and a short description of the magnetometer experiments aboard Phobos 2 were described by Riedler et al. (1989).

It is obvious from Figs 5 and 6 that the ratio of the count rates of the two HARP sensor heads in the 


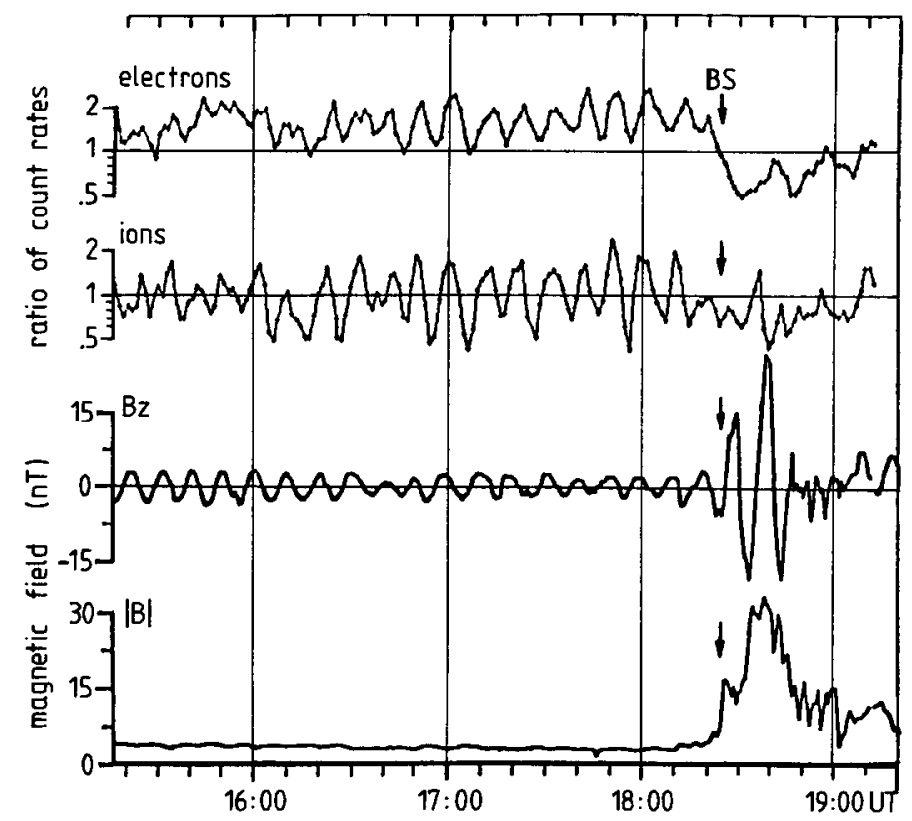

Fig. 5. RATIO OF THE COUNT RATES OF THE TWO SENSOR HEADS FOR ELECTRONS AND FOR IONS DURING THE FIRST ENCOUNTER ON 1 FEBRUARY 1989.

$B$ is the modulus of the magnetic field, $B_{z}$ is the component perpendicular to the ecliptic plane. The time of the bow shock crossing (BS) is marked.

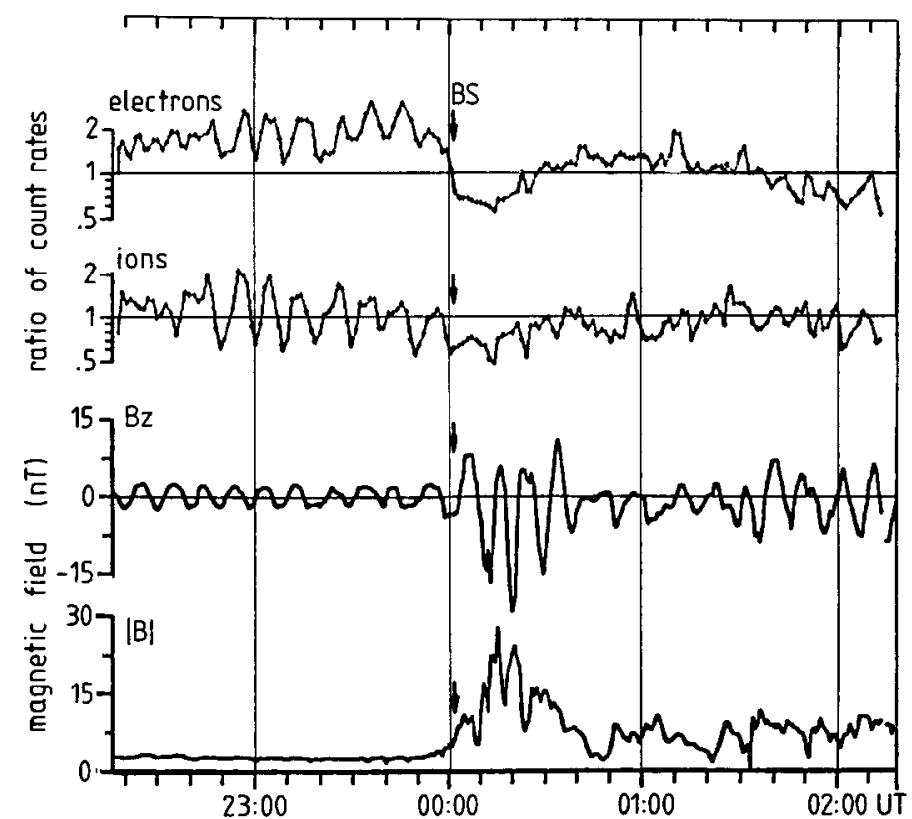

FIG. 6. RATIO OF THE COUNT RATES OF THE TWO SENSOR HEADS FOR ELECTRONS AND FOR IONS DURING THE SECOND ENCOUNTER ON 4/5 FEBRUARY 1989.

$B$ is the modulus of the magnetic field, $B_{z}$ is the component perpendicular to the ecliptic plane. The time of the bow shock crossing (BS) is marked. 
upstream region really varies at the rotation frequency both for electrons and ions for both encounters. The variation is quite regular for more than an hour preceding the bow shock crossing. Before that, double frequency variations are sometimes predominant. The amplitudes of variations are larger for ions than for electrons.

At the present stage, we do not want to jump to the conclusion that a large anisotropy of the electron and ion distributions has been detected. Alternative explanations might invoke the difference between the rotation axis and the solar wind direction, or the asymmetry of the shape and/or outside field (including the wake) of the spacecraft. We hope that better comparisons with data sets obtained by other plasma instruments will shed light on this phenomenon.

\section{CONCLUSIONS}

The HARP hyperbolic electrostatic analyzer aboard the Phobos 2 spacecraft collected important data during the high bit-rate telemetry sessions of the first and second Mars encounters. Time sequences of total count rates, ratios of count rates in different directions and energy spectra each showed a lot of structure. In this paper, the observations were mainly described and not interpreted, a detailed interpre- tation must be based on the combined set of data of all the plasma and field measurements on-board the spacecraft.

Acknowledgements-The authors wish to express their gratitude to Drs R. Grard and K. Schwingenschuh for supplying some of their unpublished data.

\section{REFERENCES}

Dubinin, E. M. et al (1990) Indirect evidences for a dust/gas torus along the Phobus orbit. Geophys. Res. Lett. 17, 861.

Grard, R. (1989) Private communication.

Grard, R. et al. (1989) First measurements of plasina waves near Mars. Nature 341, 607.

Riedler, W. et al. (1989) Magnetic fields near Mars: first results. Nature 341, 604.

Sagdeev, R. Z. and Zakharov, A. V. (1989) Brief history of the Phobos mission. Nature 341, 581.

Sagdeev, R. Z. et al. (1990) Wave activity in the neighborhood of the bow shock of Mars. Geophys. Res. Lett. 17, 893 .

Schwingenschuh, K. (1989) Private communication.

Shutte, N. M., Kiraly, P., Cravens, T. E., Dyachkov, A. V., Gombosi, T. I. Gringauz, K. I., Nagy, A. F., Sharp, W. F., Sheronova, S. M., Szegö, K., Szemerey, I., Szücs, I. T., Tatrallyay, M. and Verigin, M. I. (1989) Observation of electron and ion fluxes in the vicinity of Mars with the HARP spectrometer. Nature 341, 614 .

Shutte, N. M. et al. (1991) Energy distribution of electrons with $E<800 \mathrm{eV}$ in the areomagnetosphere. Planet. Space Sci. 39, 147.

Szücs, I. T. et al. (1990) The HARP electron and ion sensor on the Phobos mission. Nucl. Instrum. Meth. A290, 228. 